\title{
Cauchy Horizon Stability and Cosmic Censorship
}

\author{
Wenceslao S. German and Ian G. Moss \\ Department of Physics, University of Newcastle Upon Tyne, NE1 7RU U.K.
}

(March 2001)

\begin{abstract}
Some interesting consequences of spacelike matter shells are presented, in particular the possibility of travelling through Cauchy horizons and violating the strong cosmic censorship hypothesis. These show that the weak energy condition does not guarentee cosmic censorship.
\end{abstract}

Pacs numbers: 04.20.Dw 04.70.Bw

Typeset using REVTEX 
The ability to predict the future from given initial conditions might seem like an essential requirement for a classical theory of physics, but this remains an open issue in General Relativity whilst the conditions needed for cosmic censorship are not known. The situation could be resolved by finding some property of realistic matter which prevents the formation of naked singularities. In this letter we examine this question by looking at violations of cosmic censorship inside black holes.

The form of the strong cosmic censorship conjecture which we use is that 'every generic, inextendible space-time containing physically reasonable matter is globally hyperbolic' [2]. This form of the conjecture seems to be difficult to violate. If we take the charged black hole solution, for example, this is extendable beyond the globally hyperbolic region by the usual coordinate construction, but it is not considered generic because the Cauchy horizon is not stable to linear perturbations.

The instability of the Cauchy horizon has a simple physical explanation originally due to Penrose [1]. Incoming radiation ariving at the Cauchy horizon is blue shifted and the energy flux measured by an observer approaching the Cauchy horizon diverges [3, [4].

A detailed analysis of black hole perturbations in spacetimes with a cosmological constant has shown that the stability of the Cauchy horizon can still be related to the energy flux. Stability depends on the values of the surface gravity at the Cauchy horizon $\kappa_{1}$, the event horizon $\kappa_{2}$ and the cosmological horizon $\kappa_{3}$. Stability requires $\kappa_{1}<\kappa_{3}$ and $\kappa_{1}<\kappa_{2}$. [5 9 . (The second requirement was not appreciated before reference [9]).

None of the vacuum black hole spacetimes satisfy the stability requirements. We will consider the effect of adding matter, specifically spacelike shells, to the inside of the black hole. These spacelike shells are best thought of as transition layers separating different vacuum phases, where the phase transition is triggered by the high spacetime curvature inside the black hole [10]. The shells can have internal stresses and carry currents, and generalise the bubble walls associated with broken symmetry phase transitions [11].

Similar forms of matter have appeared in the literature previously in connection with the limiting curvature hypothesis, the idea that quantum gravity effects may prevent spacetime curvature singularities [12]. The spacelike shells allow the transformation of the black hole into a nonsingular wormhole. However, we shall consider only black holes with singularities.

The spacetime is shown in figure (1). The shell is placed at $r_{s}$ and the metric on either side of the shell is given by

$$
d s^{2}=-\frac{r^{2}}{\Delta} d r^{2}+\frac{\Delta}{r^{2}} d t^{2}+r^{2} \Omega^{2}
$$

where

$$
\Delta=2 M r-r^{2}+Q^{2}-\frac{1}{3} \Lambda r^{4}
$$

For $r<r_{s}$, the parameters take on values $M_{1}, Q_{1}$ and $\Lambda_{1}$ and for $r>r_{s}, M_{2}, Q_{2}$ and $\Lambda_{2}$. Both $\Lambda_{1}$ and $\Lambda_{2}$ will be suposed fixed by the details of some phase transition. For simplicity, we will take $Q_{2}=0$. This would be the case, for example, if the charge $Q_{1}$ is associated with a gauge symmetry which is broken outside the shell. 


\section{FIGURES}

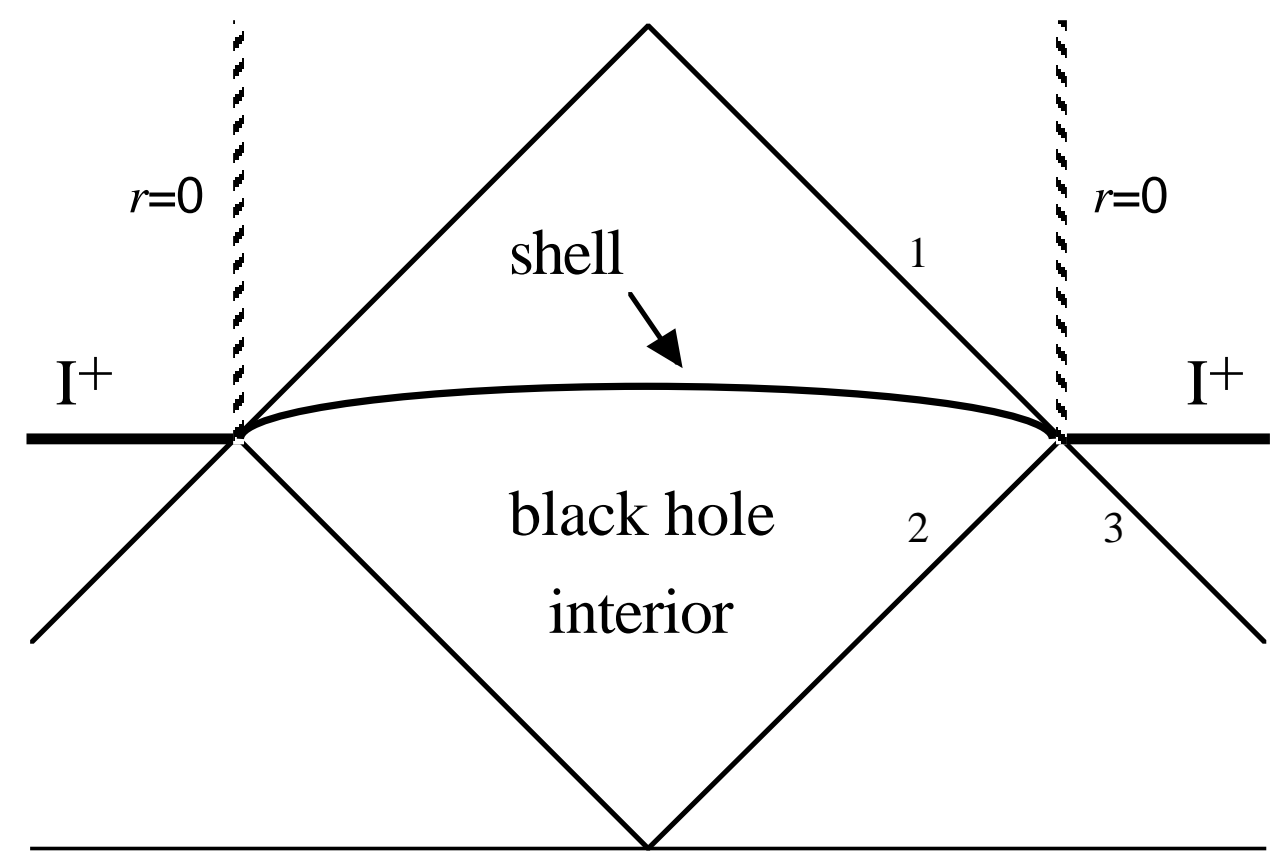

FIG. 1. Penrose diagram of the charged black hole with inner horizon $r=r_{1}$, event horizon $r=r_{2}$ and cosmological horizon $r=r_{3}$. The shell lies between the inner and the event horizons.

Suppose that $h_{a b}$ is the metric induced in the shell and $u_{a}$ is a unit vector along the $t$ direction, then the stress-energy of the shell has the form 12,13

$$
S_{a b}=p_{s} u_{a} u_{b}+p_{\perp}\left(h_{a b}-u_{a} u_{b}\right) .
$$

The junction conditions imply that the metric is continuous and the extrinsic curvature satisfies,

$$
\left[K_{a b}\right]=-8 \pi\left(S_{a b}-\frac{1}{2} h_{a b} S\right)
$$

For the metric (1),

$$
\begin{aligned}
{\left[\Delta^{1 / 2}\right] } & =4 \pi r^{2} p_{s} \\
{\left[r\left(\Delta^{1 / 2}\right)^{\prime}\right] } & =8 \pi r^{2} p_{\perp} .
\end{aligned}
$$

The junction conditions can be solved for $M_{1}$ and $Q_{1}$ as functions of the radius $r_{2}$ and the pressures $p_{s}$ and $p_{\perp}$. Figure (2) shows contours of constant pressure $p_{\perp}$ when $p_{s}=0$. As $p_{\perp} \rightarrow \infty$, the solution approaches a line

$$
Q^{2}-Q_{c}^{2}=2 r_{2}\left(M-M_{c}\right)
$$

where $M_{c}=r_{2}-\frac{2}{3} \Lambda_{1} r_{2}^{2}$ and $Q_{c}^{2}=r_{2}^{2}-\Lambda r_{2}^{4}$ correspond to a black hole with coincident horizons. 


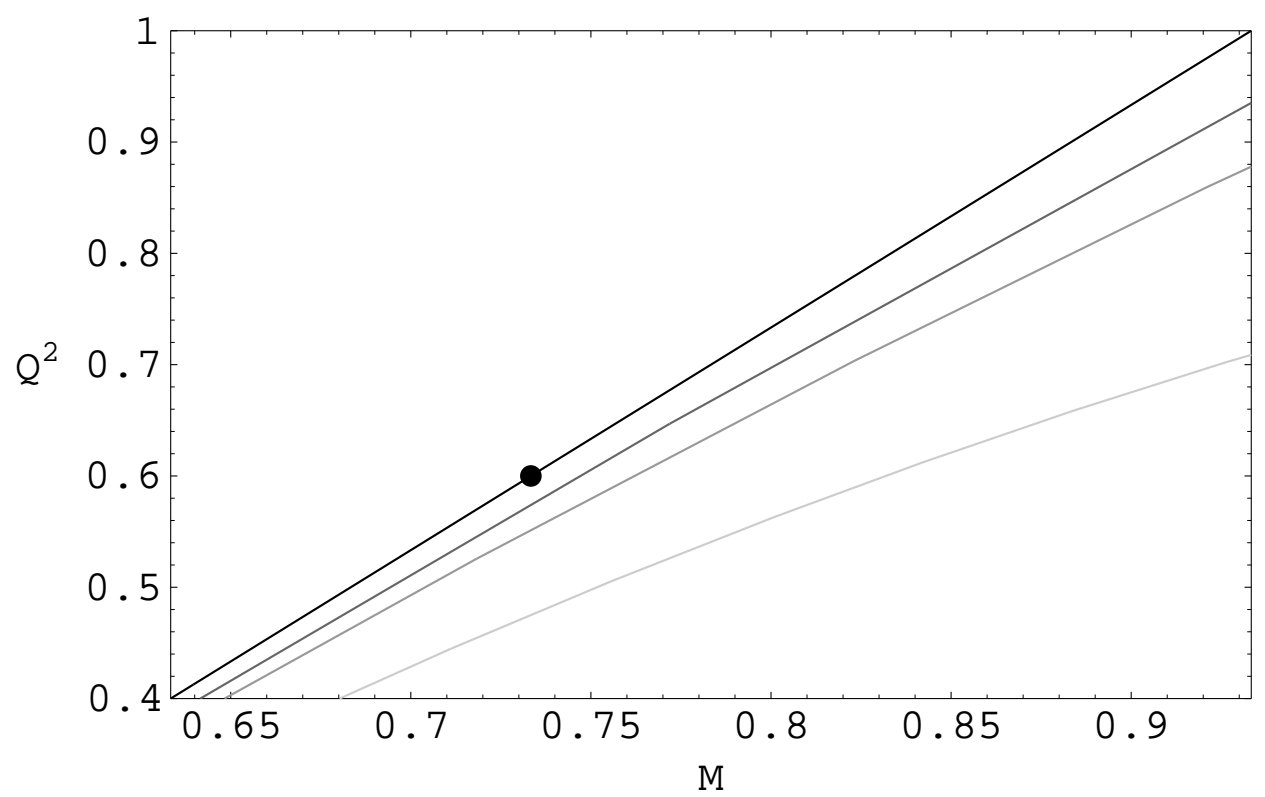

FIG. 2. Contours of constant shell pressure $p_{\perp}$ have been drawn on the charge and mass paramater space of the inner black hole solution. Lighter gray indicates smaller pressure. The straight line is the limiting case $p_{\perp} \rightarrow \infty$. (the parameters are scaled by the horizon radius, $M=M_{1} / r_{2}, Q=Q_{1} / r_{2}$ and $\left.\Lambda_{1} r_{2}^{2}=0.4\right)$

The linear perturbation analysis of the black hole can be carried out on either side of the shell. The perturbations propagate as waves and undergo a constant redshift at the shell, leaving the same Cauchy horizon stabilty conditions on the surface gravity as before.

The Cauchy horizon lies inside the shell at $r=r_{1}$, where the surface gravity is given by $\kappa_{1}=\Delta^{\prime} /\left(2 r^{2}\right)$. From (2), and the condition $\Delta\left(r_{1}\right)=0$, one can obtain

$$
\begin{aligned}
M_{1} & =\kappa_{1} r_{1}^{2}+r_{1}-\frac{2}{3} \Lambda_{1} r_{1}^{3} \\
Q_{1}^{2} & =2 \kappa_{1} r_{1}^{3}+r_{1}^{2}-\Lambda_{1} r_{1}^{4}
\end{aligned}
$$

Figure (3) shows contours of constant $\kappa_{1}$ in the $\left(M_{1}, Q_{1}\right)$ parameter space. The surface gravity vanishes at the point $\left(M_{c}, Q_{c}\right)$. Comparing this figure with figure (2), we see that if the pressure is sufficiently large, we can make the surface gravity arbitrarily small. In particular, we can satisfy the stability requirements $\kappa_{1}<\kappa_{2}$ and $\kappa_{1}<\kappa_{3}$ for the Cauchy horizon. 


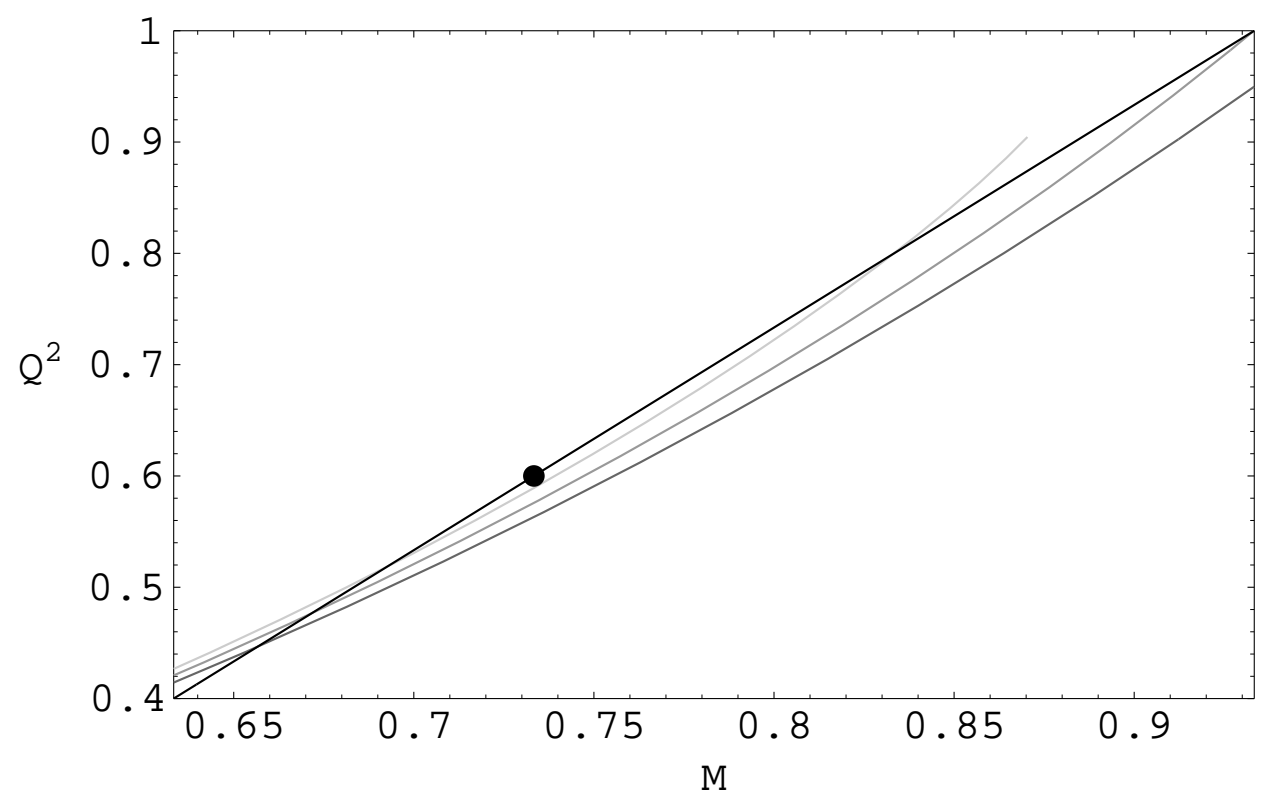

FIG. 3. Contours of constant surface gravity $\kappa_{1}$ have been drawn on the charge and mass parameter space of the inner black hole solution. The straight line is the limiting case of the shell pressure $p_{\perp} \rightarrow \infty$.

The stability of the shell itself can be analysed by taking the radius $r_{s}$ to be a function of the proper time along the shell, as described in reference [13. The junction conditions become

$$
\begin{aligned}
{\left[K_{\theta \theta}\right] } & =4 \pi r^{2} p_{s} \\
{\left[\dot{K}_{\theta \theta}\right] } & =8 \pi r \dot{r} p_{\perp}
\end{aligned}
$$

where $K_{\theta \theta}=\left(r^{2} \dot{r}^{2}+\Delta\right)^{1 / 2}$. If $\delta p_{s}=c^{2} \delta p_{\perp}$, perturbations about the static shell satisfy

$$
\left[\Delta^{-1 / 2}\right] \delta \ddot{r}+\left(\frac{1}{2}\left[r^{-1}\left(\Delta^{-1 / 2} r^{-1} \Delta^{\prime}\right)^{\prime}\right]-\frac{2}{c^{2}}\left[r^{-1}\left(r^{-2} \Delta^{1 / 2}\right)^{\prime}\right]\right) \delta r=0
$$

We find neutral stability when $p_{s}=0$, whilst for $0 \leq p_{s} \ll p_{\perp}$ there exists a $c_{\max }$ such that stability occurs for $0<c<c_{\max }$.

The spacelike shells which we have considered have zero density and therefore when $p_{s} \geq 0$ they marginally satisfy the weak energy condition, $\rho \geq 0$ and $\rho+p \geq 0$. We can infer that the weak energy condition does not imply strong cosmic censorship.

The spacelike shells considered by Frolov et al. [12] were specifically aimed at domonstrating the possibility of singularity avoidance in gravitational collapse by the creation of wormholes and for this the shell must have 'exotic' matter with $p+\rho<0$. Wormholes are not globally hyperbolic and violate the strong cosmic censoship principle. We find that spacelike shells which satisfy the weak energy condition can still violate cosmic censorship and produce naked singularities. 


\section{ACKNOWLEDGMENTS}

We would like to thank Manolo Per for helpful discussions. WSG is supported by CONACYT (Mexico) grant number 116020. 


\section{REFERENCES}

[1] Penrose R 1969 Gravitational Collapse: the role of General Relativity, Riv. del Nuove Cimento 1

[2] Clarke C J S 1993 The analysis of spacetime singularities (Cambridge University Press)

[3] Chandrasekhar S and Hartle J B 1982 Proc. R. Soc. 484301

[4] Chandrasekhar S 1983 The mathematical theory of black holes (Cambridge University Press)

[5] Mellor F A and Moss I G 1990 Phys. Rev. D 41 403; 1992 Class. Quantum Grav. 9 L43

[6] Brady P R and Poisson E 1992 Class. Quantum Grav. 9121

[7] Chambers C M and Moss I G 1994 Class. Quantum Grav. 111035

[8] Brady P R, Nuñez D and Sinha S 1993 Phys. Rev. D 47

[9] Brady P R, Moss I G and Myers R 1998 Phys. Rev. Lett. 803432

[10] Shore G M 1980 Ann. Phys. 128376

[11] Blau S K, Guendelman E I and Guth A H 1987 Phys. Rev. D 351747

[12] Frolov V P, Markov M A and Mukhavov V F 1989 Phys. Lett. B216 272; 1990 Phys. Rev. D 41383

[13] Balbinot B and Poisson E 1990 Phys. Rev. D 41395 